\title{
Pharmabook - Um Ambiente Colaborativo para Pesquisa de Preços e Controle de Estoque Pessoal de Medicamentos
}

\author{
Rafael V. B. Ferreira, Irlon S. Lamblet, Carolina L. Aguilar, \\ Luis C. B. Silva, Rafael E. L. Escalfoni \\ Centro Federal de Educação Tecnológica Celso Suckow da Fonseca (CEFET-RJ) \\ Av. Gov. Roberto Silveira, 1900, Prado - Nova Friburgo - RJ - Brasil \\ \{rafaelviniciusbarros5, irloncefet\}@gmail.com, \{carolina.aguilar, \\ luis.silva, rafael.escalfoni\}@cefet-rj.br
}

\begin{abstract}
Access to drugs is a fundamental part of improving the population's quality of life. With the improve of the conditions of treatments and increase of life expectation, the medicine consumption has increased, representing an item with the highly impact on the domestic budget. This paper presents the Pharmabook, a collaborative environment for price sharing aimed at facilitating access to information on drug prices, as well as to help control the personal stock of medications, aiming at better planning of purchases.
\end{abstract}

Resumo. $O$ acesso a medicamentos é parte fundamental para melhoria da qualidade de vida das populações. Com o aprimoramento das condições de tratamentos e o aumento da expectativa de vida, o consumo de medicamentos tem crescido, constituindo um dos itens de maior impacto no orçamento doméstico. $O$ presente artigo apresenta o Pharmabook, um ambiente colaborativo que visa facilitar o acesso a informação sobre preços de medicamentos, como também, auxiliar no controle do estoque pessoal de medicamentos, visando um melhor planejamento das compras.

\section{Introdução}

Os medicamentos constituem parte essencial para a promoção da melhoria da qualidade de vida das populações e para o sucesso de ações promovidas pelo sistema de saúde. A melhoria da qualidade de tratamentos e o aumento da expectativa de vida da população tem feito crescer o consumo de medicamentos.

Estima-se que no Brasil, o consumo de medicamentos constitua um dos itens de maior gasto familiar [Crispin 2008]. A discrepância de valores oferecidos no varejo tem forte influência nos gastos, muito por conta da assimetria de informações e da fidelidade às marcas, apesar da ampla divulgação dos medicamentos genéricos promovida pela Política Nacional dos Medicamentos desde 1998 [Miranda et al. 2009].

O presente trabalho apresenta o Pharmabook, um aplicativo que dispõe de mecanismos de colaboração entre usuários para divulgação de preços praticados por diferentes farmácias na vizinhança e um controle de estoque para que o consumidor possa planejar suas compras. O restante do artigo está estruturado como segue: na Seção 2, é apresentado o referencial teórico, enquanto na Seção 3 é resumido o aplicativo Pharmabook. Os resultados obtidos são apresentados na Seção 4 e, por último, na Seção 5 são feitas as conclusões finais. 


\section{Referencial Teórico}

Nas últimas décadas, o Brasil tem ampliado suas políticas públicas na tentativa de oferecer a população maior acesso a medicamentos e atendimento de saúde. Seguindo uma tendência de outros países, o governo brasileiro promoveu em 1998 uma política de regulamentação de medicamentos genéricos, para estimular a concorrência comercial e melhorar a qualidade dos medicamentos [Miranda et al. 2009].

Com um número excessivo de farmácias no Brasil, estima-se uma razão de uma loja para cada 3.500 habitantes [Silva e Rocha 2016], as farmácias recorrem a artifícios como vender medicamentos bonificados para se manterem abertas. A indicação de tais medicamentos, unida à falta de conhecimento do consumidor, tem prejudicado a venda de genéricos e impactado no preço final ao consumidor.

Um outro importante aspecto é o uso racional dos medicamentos, que segundo a OMS (2002), ocorre quando os pacientes recebem medicação apropriada às suas necessidades clínicas, dentro de parâmetros de dosagem e período recomendados para seu quadro e ao menor custo possível para si e para a comunidade [Aquino 2008]. Ocorre que no Brasil, muitas vezes a população estoca medicamentos em casa de maneira inapropriada, o que leva a inúmeros casos de intoxicações medicamentosas [Mastroianni et al. 2011].

Tais problemas se acentuam nos casos de pessoas portadoras de doenças crônicas e idosos, devido ao uso habitual de medicamentos e, normalmente, não podem deixar de usá-los. Apesar do Sistema Único de Saúde (SUS) fornecer gratuitamente uma relação de medicamentos essenciais, muitos medicamentos de uso contínuo estão fora da lista [Helfer et al. 2012]. Paniz e sua equipe (2008), em estudo feito em municípios do Sul e Nordeste constataram que o acesso a medicamentos contínuos em adultos chega a $81 \%$, logo, o custo da medicação e sua correta estocagem é fundamental para o equilíbrio das contas domésticas. O Pharmabook foi concebido para lidar com tais dificuldades, buscando reduzir os impactos no orçamento familiar.

\section{Projeto do Pharmabook}

Para o método de desenvolvimento adotou-se como base o Lean Startup, no qual prega a construção de um produto mínimo viável (MVP - Minimum Viable Product), visando a criação de um produto a partir do que realmente importa ao cliente [Ries 2011].

Como ferramenta para especificação dos requisitos funcionais do sistema foi utilizada a UML, com foco na representação das funcionalidades, estrutura e interação das classes dos objetos do sistema. Dentre os modelos desenvolvidos, a Figura 1 apresenta do diagrama de casos de uso com as principais funcionalidade do aplicativo.

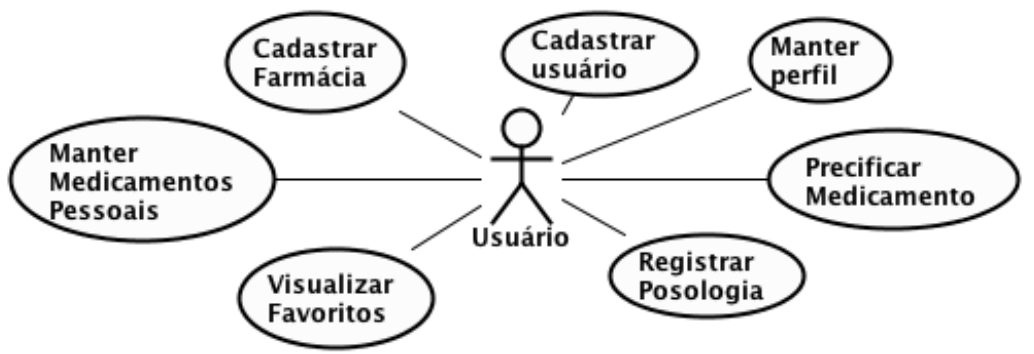

Figura 1. Casos de Uso da aplicação Pharmabook 
Quadro 1: Descrição resumida dos Casos de Uso do aplicativo PharmaBook

\begin{tabular}{|l|l|}
\hline \multicolumn{1}{|c|}{ Tema } & \multicolumn{1}{c|}{ Funcionalidades } \\
\hline $\begin{array}{l}\text { Precificar } \\
\text { Medicamento }\end{array}$ & $\begin{array}{l}\text { O usuário pode atualizar os preços dos medicamentos consultados nas } \\
\text { farmácias, estes são imediatamente disponibilizados para a comunidade de } \\
\text { usuários do Pharmabook. }\end{array}$ \\
\hline Registrar Posologia & $\begin{array}{l}\text { Registro da periodicidade para uso de cada medicamento registrado no } \\
\text { aplicativo para uso pessoal. Utilizado para contabilização do estoque. }\end{array}$ \\
\hline Visualizar Favoritos & $\begin{array}{l}\text { Recurso de consulta rápida dos medicamentos precificados selecionados } \\
\text { como favoritos na base pessoal do usuário. }\end{array}$ \\
\hline $\begin{array}{l}\text { Manter Medicamentos } \\
\text { Pessoais }\end{array}$ & $\begin{array}{l}\text { Permite o cadastro dos medicamentos utilizados pelo usuário. Utiliza como } \\
\text { apoio base de medicamentos disponibilizada pela Anvisa }\end{array}$ \\
\hline Manter Farmácia & $\begin{array}{l}\text { Permite o cadastro das farmácias pelo usuário, que ficam disponíveis para a } \\
\text { comunidade de usuários do aplicativo. }\end{array}$ \\
\hline Cadastrar Usuário & Permite o cadastro de novos usuário para uso do aplicativo. \\
\hline Manter Perfil & $\begin{array}{l}\text { Permite que o usuário faça atualizações dos dados do seu perfil, alteração do } \\
\text { login e senha , como também, a exclusão do perfil. }\end{array}$ \\
\hline
\end{tabular}

Para o projeto arquitetural da aplicação, foi utilizado a estrutura de camadas do padrão Model-View-Controller (MVC), visando trazer ao projeto uma boa separação da interface de usuário, lógica de negócios e separação de responsabilidades. Em relação à codificação do lado servidor, foi utilizada a linguagem PHP com o framework de desenvolvimento Slim. No lado do cliente, foram utilizadas tecnologias como, HTML, CSS, Bootstrap, Datatable, JavaScript junto de bibliotecas jQuery e jQuery mobile. O SGBD usado foi o MySQL unido ao ambiente de desenvolvimento do Xampp.

\section{Resultados Obtidos}

O design responsivo aplicado no desenvolvimento da interface do usuário propiciou um uso adequado em dispositivos móveis, o que aumenta a aderência ao uso do aplicativo. Também, análise de interação humano-computador foram realizadas visando que o aplicativo pudesse oferecer boa acessibilidade para pessoas idosas. A Figura 2 apresenta o fluxo de telas ao monitorar e detalhar preços de um medicamento.

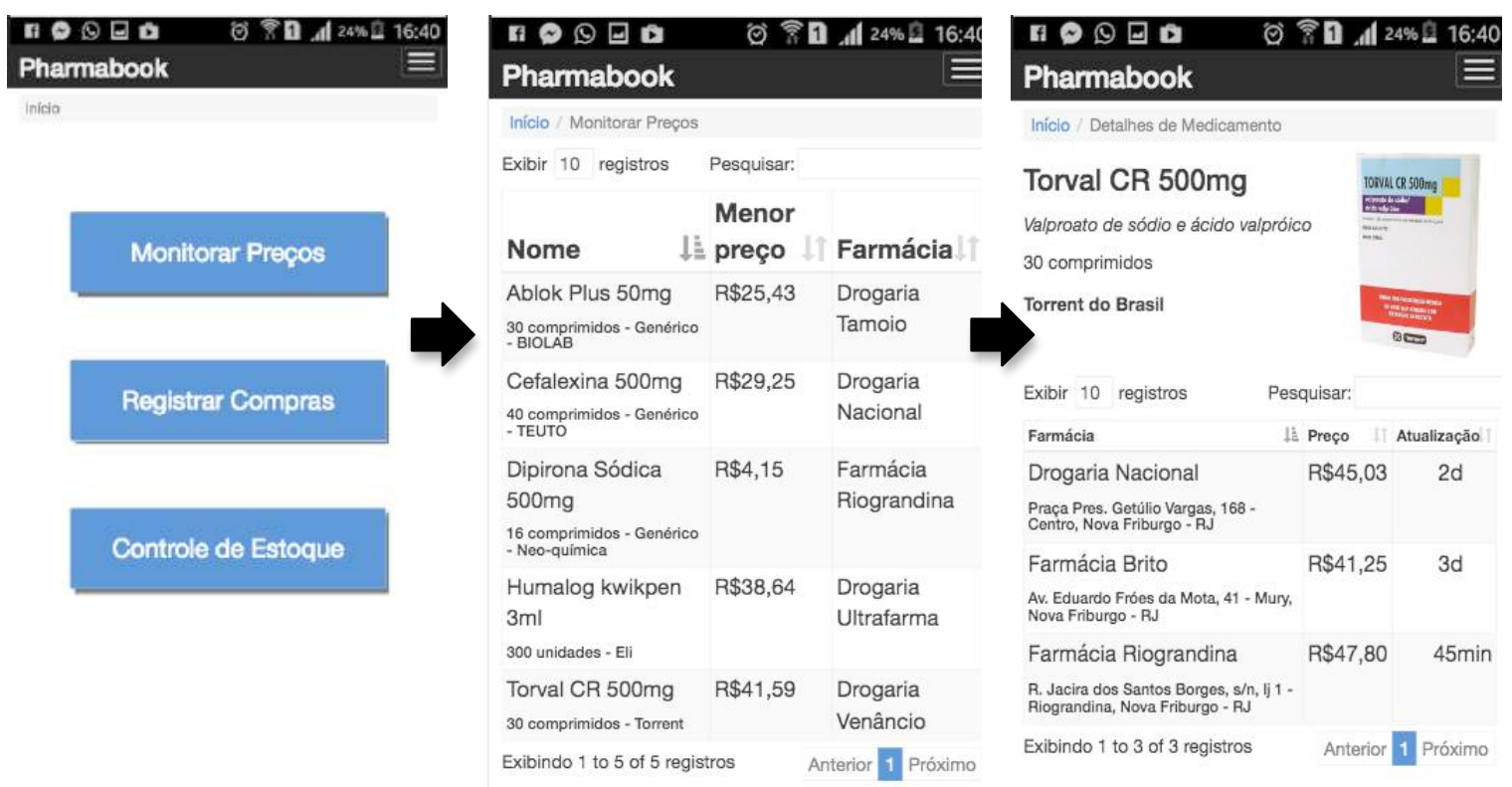

Figura 2. Telas do aplicativo Pharmabook 


\section{Conclusão e trabalhos futuros}

O preço de medicamentos sofre com uma alta variabilidade entre farmácias e impacta no orçamento doméstico. Possibilitar o acesso a informações sobre o valor médio e a disponibilidade do produto nas lojas próximas pode auxiliar na decisão de compra, reduzindo seus custos. Diante desse cenário, e por conta da popularização dos aparelhos smartphones, apresentamos neste trabalho o Pharmabook, um aplicativo para controle de estoque de medicamentos e busca de preços. Desenvolvido com uso da metodologia Lean Startup que proporciona interações rápidas com futuros usuários, o sistema utiliza tecnologias responsivas independentes de plataforma. Futuramente, pretendemos rodar um experimento formal para validar a eficácia da ferramenta e evoluir os ciclos de interação para acrescentar novas funcionalidades.

\section{References}

Aquino, D. S. de (2008), "Por que o uso racional de medicamentos deve ser uma prioridade?", In Ciência \& Saúde Coletiva vol. 13, pag. 733-736.

Crispin, R. I. C. (2008), "Impacto da Política Nacional de Medicamentos (PNM) no Acesso a Medicamentos Gratuitos”, Dissertação - UFC, Fortaleza, CE.

Helfer A. P., Camargo A. L., Tavares N. U. L., Kanavos P., and Bertoldi A. D. (2012), "Capacidade aquisitiva e disponibilidade de medicamentos para doenças crônicas no setor public", In Rev Panam Salud Publica, 2012, vol. 31(3), pag. 225-232.

Mastroianni P. C., Lucchetta R. C., Sarra J. R., and Galduróz J. C. F. (2011), "Estoque doméstico e uso de medicamentos em uma população cadastrada na estratégia saúde da família no Brasil”, In Rev Panam Salud Publica. 2011, vol. 29(5), pag. 358-64.

Miranda, E. S., Pinto, C. B. S., Reis, A. L. A., Emmerick, I. C. M., Campos, M. R., Luiza, V. L., e Osorio-de-Castro, C. G. S. (2009), "Disponibilidade no setor público e preços no setor privado: um perfil de medicamentos genéricos em diferentes regiões do Brasil”, In Caderno de Saúde Pública, 2009, vol. 25(10), pag. 2147-2158.

OMS - Organización Mundial de la Salud. (2002), "Perspectivas políticas sobre medicamentos de la OMS. Promoción del uso racional de medicamentos: componentes centrales", Disponível em: <http://apps.who.int/medicinedocs/pdf/ s4874s.pdf>. Acessado em 11 fev 2017.

Paniz, V. M. V., Fassa, A. G., Facchini, L. A., Bertoldi, A. D., Piccini, R. X., Tomasil, E., Thumé, E., Silveira, D. S., Siqueira, F. V., and Rodrigues, M. A. (2008), “Acesso a medicamentos de uso contínuo em adultos e idosos nas regiões Sul e Nordeste do Brasil”, In Cad. Saúde Pública, Rio de Janeiro, vol. 24(2), Feb. 2008.

Ries, E. (2011) “The lean startup: How today's entrepreneurs use continuous innovation to create radically successful businesses", Crown Business, 2011.

Silva, N. C. S. and Rocha, L. C. R. (2016), "Medicamentos Genéricos: Legislação, Política e Mercado", In ÚNICA Cadernos Acadêmicos, vol. 3(1) (2016). 